\title{
Terahertz conductivity of thin metal films
}

\author{
N. Laman and D. Grischkowsky \\ School of Electrical and Computer Engineering, Oklahoma State University, Stillwater, \\ Oklahoma 74078, USA
}

(Received 19 March 2008; accepted 17 July 2008; published online 5 August 2008)

\begin{abstract}
The conductivities of thin $\mathrm{Al}, \mathrm{Au}$, and $\mathrm{Ag}$ films were measured via their transmission at terahertz frequencies. The conductivities of all the films, particularly the thinner films and Al films, were much smaller than their bulk dc values. This reduced conductivity can be quantitatively understood in terms of an increased scattering rate from defects. The transmission is consistent with a frequency independent conductivity, implying a very fast electron scattering time. (C) 2008 American Institute of Physics. [DOI: 10.1063/1.2968308]
\end{abstract}

The conductivity of metals at terahertz $(\mathrm{THz})$ frequencies is becoming increasingly important as both microcircuit technology and microwave resonators are being driven at ever-higher frequencies. Our earlier work with terahertz metal parallel-plate waveguides ${ }^{1}$ has shown that the conductivity of $\mathrm{Al}$ and $\mathrm{Cu}$ at $\mathrm{THz}$ frequencies is considerably less than the expected handbook value, particularly at cryogenic temperatures, due to defects within the $100 \mathrm{~nm}$ skin-depth layer. We have now extended and confirmed these results by measuring the terahertz transmission of a number of thin films comprised of different metals and different thicknesses. Unlike our previous work, this allows us to measure the conductivity of the thin film as a function of the thickness, since we are probing the entire film and not just the skin-depth layer.

Thin films of $\mathrm{Al}, \mathrm{Au}$, and $\mathrm{Ag}$ were deposited on highresistivity, 2 in. diameter, $0.43 \mathrm{~mm}$ thick $\mathrm{Si}$ wafers via thermal evaporation. The typical evaporation rates were $2 \mathrm{~nm} / \mathrm{s}$ onto an unheated wafer in order to ensure a relatively high conductivity film. The thickness of the film was measured via a quartz thickness monitor. Six films were made in total: Al films of 36, 88, and $152 \mathrm{~nm}$ thickness, Au films of 85 and $150 \mathrm{~nm}$ thickness, and an Ag film of $86 \mathrm{~nm}$ thickness. The films were not annealed after evaporation. The metal coated wafers were placed in a standard terahertz time-domain spectroscopy (THz-TDS) apparatus. ${ }^{2,3}$ Their transmission was measured at both 295 and $77 \mathrm{~K}$. The terahertz pulses and their amplitude spectra are shown in Fig. 1.

The amplitude transmission at a frequency $f$ of the films and wafers can be modeled by the thin film formula ${ }^{4}$

$$
t=\frac{t_{12} t_{23} t_{34} \exp \left[i(2 \pi h f / c) n_{2}\right] \exp \left[i(2 \pi d f / c) n_{3}\right]}{1+r_{12} r_{23} \exp \left[2 i(2 \pi h f / c) n_{2}\right]},
$$

where $t_{i j}$ and $r_{i j}$ are the complex Fresnel transmission and reflection coefficients, ${ }^{4}$ respectively, medium 1 and 4 are vacuum, medium 3 is $\mathrm{Si}$ with a thickness $d=0.43 \mathrm{~mm}$ and with a $\mathrm{THz}$ frequency independent index $n_{3}=3.4175$ and negligible terahertz absorption, ${ }^{5}$ and medium 2 is the metal film with a thickness of $h$ and a complex index ${ }^{4,6}$ of

$$
n_{2}=(1+i) \sqrt{\sigma /\left(4 \pi \varepsilon_{0} f\right)} \text {. }
$$

Propagation within the metal is described by $\exp [\mathrm{ikz}-\omega t]$ where $k=(2 \pi f / c) n_{2}$. Using Eq. (2), one obtains the ampli-

${ }^{a)}$ Electronic mail: daniel.grischkowsky@okstate.edu. tude absorption coefficient $\alpha$ to be $\alpha=\operatorname{Im}(k)=1 / \delta=\sqrt{\pi f \sigma \mu_{0}}$. Note that $\alpha$ is simply $1 / \delta$, where $\delta$ is the usual result for the skin depth. ${ }^{6,7}$

For our case of a $88 \mathrm{~nm} \mathrm{Al} \mathrm{film} \mathrm{with} \mathrm{a} \mathrm{conductivity} \mathrm{of}$ $\sigma=16(\mu \Omega)^{-1}, n_{2}=(1+i) 370$ at $1 \mathrm{THz}$, the resulting amplitude attenuation traversing a single pass within the metal film [i.e., $\exp (-\alpha z)]$ is only $49 \%$, illustrating that the low transmission of these films is dominated by the high reflections from surfaces 1,2 and 2,3. Given the high reflectivity of these metal films, the amplitude transmission $t$ tended to be low, on the order of $10^{-2}-10^{-3}$, corresponding to a power transmission on the order of $10^{-4}-10^{-6}$.

The measured amplitude transmissions $t$ of the $88 \mathrm{~nm} \mathrm{Al}$ film, the $85 \mathrm{~nm}$ Au film, and the $86 \mathrm{~nm} \mathrm{Ag} \mathrm{film} \mathrm{are} \mathrm{shown} \mathrm{in}$ Figs. 2-4, along with the theoretical curves [from Eqs. (1) and (2)] and for which the frequency independent conductivity was adjusted for the best fit. The fitted conductivities are shown in Table I. The conductivity of all the films is considerably less than the handbook ${ }^{8}$ bulk dc value. In general, the conductivity is less for thinner films, particularly at $77 \mathrm{~K}$. Films with higher percentage conductivity relative to the bulk conductivity at $295 \mathrm{~K}$ showed a larger relative conductivity increase at lower temperature. This would be consistent with the films having additional, temperature independent electron scattering mechanisms compared to the bulk,
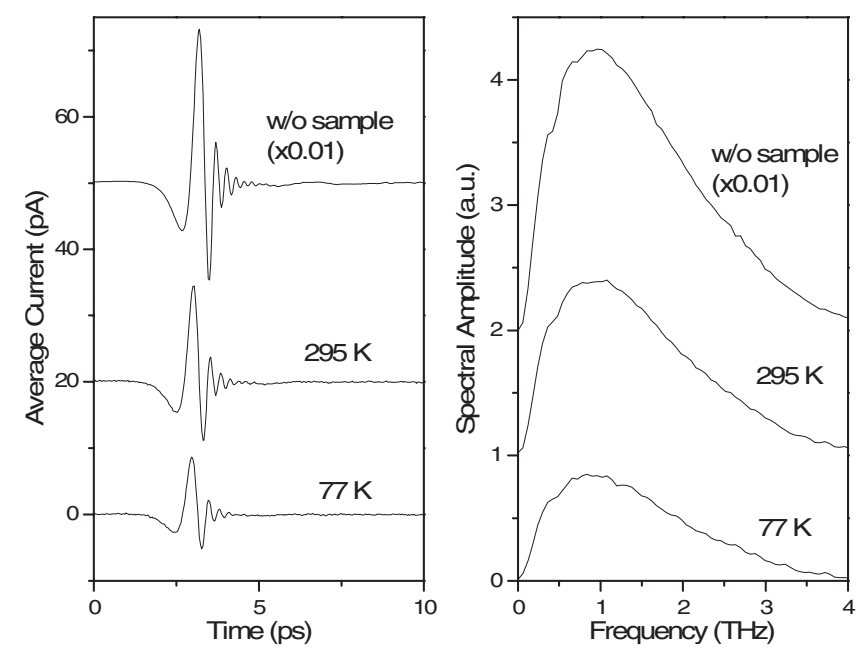

FIG. 1. Terahertz pulses through $88 \mathrm{~nm} \mathrm{Al} \mathrm{film} \mathrm{and} \mathrm{corresponding} \mathrm{spectra}$ Signals at $295 \mathrm{~K}$ and reference signal (without sample) are offset for clarity. Reference signal is multiplied by a factor of 0.01 . 


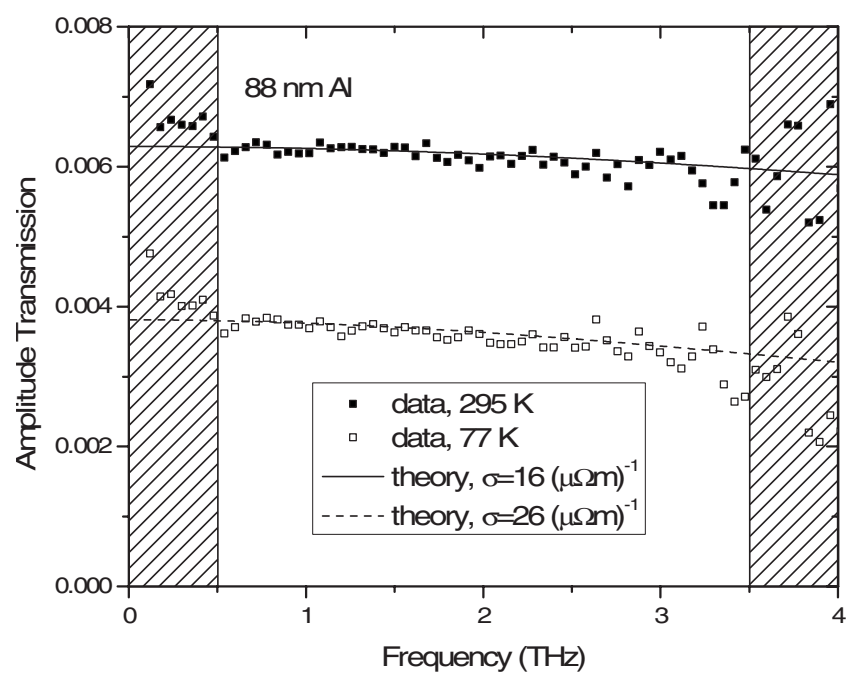

FIG. 2. Amplitude transmission $t$ through $88 \mathrm{~nm} \mathrm{Al}$ film at 295 and $77 \mathrm{~K}$. The shaded regions correspond to frequencies with lower signal to noise.

such as defects, grain boundaries, and surfaces. These mechanisms would be more prevalent in the thinner films, resulting in a lower relative conductivity.

There is also a marked difference between the different metals. The $\mathrm{Al}$ films had the lowest conductivity compared to the bulk values $(42 \%-58 \%$ at $295 \mathrm{~K}$ and $6 \%-12 \%$ at $77 \mathrm{~K})$ while the $\mathrm{Au}$ and $\mathrm{Ag}$ films had the highest (33\%-73\% at 295 $\mathrm{K}$ and $12 \%-34 \%$ at $77 \mathrm{~K})$. The $\mathrm{Ag}$ film $(86 \mathrm{~nm})$ had the additional distinction of having similar behavior to the thicker Au film at $150 \mathrm{~nm}$. This would imply that the quality is best for the Ag film and worst for the Al films.

The dependence of the conductivity of thin metal films at dc frequencies has been well studied by the four-point probe method. ${ }^{9,10}$ The dc conductivity of thin films is considerably less than the bulk conductivity and is reduced as the thickness of the film is reduced. The general consensus is that this low conductivity is due to the scattering from grain boundaries. ${ }^{9,10}$

For $\mathrm{Al}$ and $\mathrm{Au}$, the conductivity measured with the $\mathrm{dc}$ thin film experiments at $80 \mathrm{~K}$ is two to four times greater than the corresponding conductivity measured with terahertz. For $\mathrm{Ag}$, the conductivity measured by the dc thin film experiments at $80 \mathrm{~K}$ is only $10 \%$ greater than the corresponding

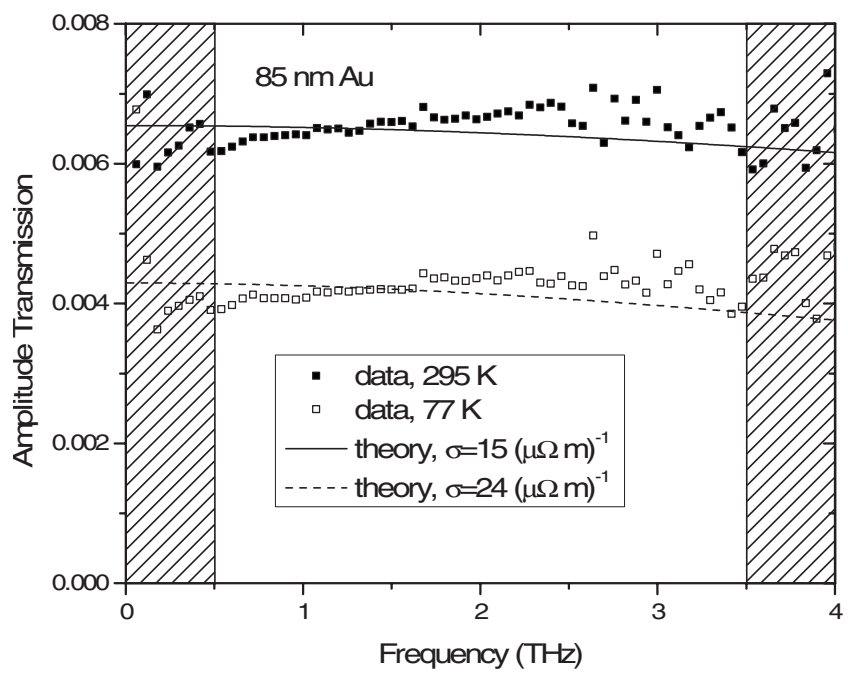

FIG. 3. Amplitude transmission $t$ through $85 \mathrm{~nm}$ Au film at 295 and $77 \mathrm{~K}$.

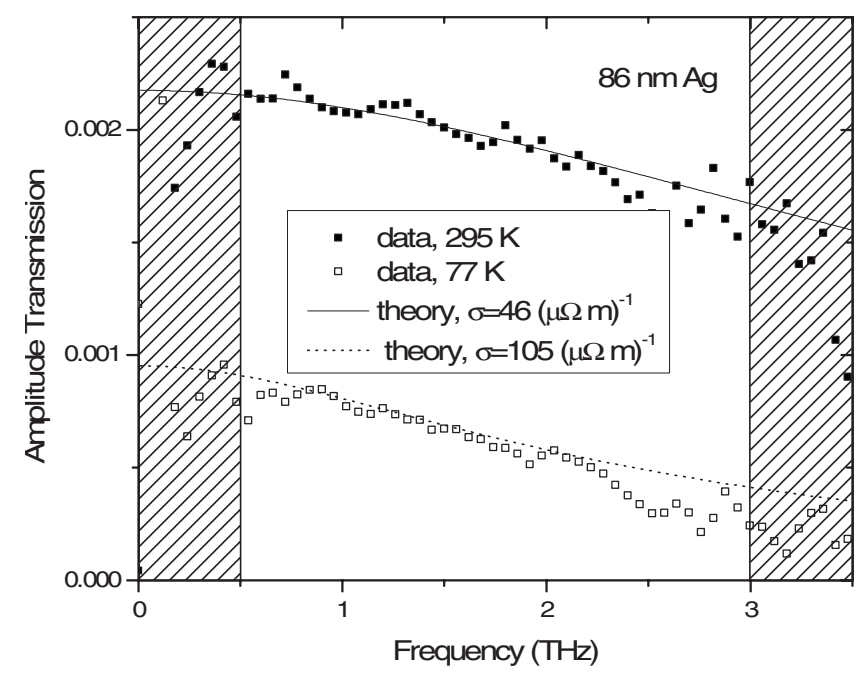

FIG. 4. Amplitude transmission $t$ through $86 \mathrm{~nm} \mathrm{Ag} \mathrm{film} \mathrm{at} 295$ and $77 \mathrm{~K}$.

terahertz measurements. This large difference between the dc and terahertz measurements may be due to different conductivities near different interfaces of the metal film. Our earlier work ${ }^{1}$ revealed that the metal near the metal/air interface has considerably smaller conductivity than the metal near the metal/Si interface. The metal in the interior of the film would be expected to have the least number of defects, and hence the highest conductivity. While the dc work samples the entire thickness in a relatively uniform manner, our terahertz measurements are particularly sensitive to the conductivity near the interfaces, as they contribute to the very large reflections.

The thicknesses of all of the films measured in this paper were all larger than the percolation transition. ${ }^{11}$ In addition, the film thicknesses are all larger than the mean free path of the electrons given either the measured conductivities or the bulk conductivities at $295 \mathrm{~K}^{12}$

For the thinner films with less conductivity, the losses in the metal film itself have only slight positive frequency dependence (i.e., the higher frequencies have a smaller skin depth, corresponding to larger losses). This is offset by the inverse frequency dependence of the losses due to the reflections, resulting in a nearly frequency independent transmission. For the thicker films with more conductivity, the frequency dependence of the losses in the metal film dominate, resulting in a smaller transmission at higher frequencies.

In general, the conductivity can also be frequency dependent. This is most simply modeled by the Drude

TABLE I. Conductivity $\left[(\mu \Omega \mathrm{m})^{-1}\right]$ of films; indicated percentage is relative to bulk conductivity from Ref. 8 .

\begin{tabular}{lccccc}
\hline \hline Metal & $\begin{array}{c}\text { Thickness } \\
(\mathrm{nm})\end{array}$ & $\sigma$ at $295 \mathrm{~K}$ & $\sigma$ at $77 \mathrm{~K}$ & $\begin{array}{c}\text { Bulk } \sigma_{\mathrm{dc}}{ }^{\mathrm{a}} \\
\text { at } 295 \mathrm{~K}\end{array}$ & $\begin{array}{c}\text { Bulk } \sigma_{\mathrm{dc}}{ }^{\mathrm{a}} \\
\text { at } 80 \mathrm{~K}\end{array}$ \\
\hline & 36 & $17(45 \%)$ & $25(6 \%)$ & & \\
& 88 & $16(42 \%)$ & $26(6 \%)$ & & 408 \\
$\mathrm{Al}$ & 152 & $22(58 \%)$ & $51(12 \%)$ & 37.7 & \\
& 85 & $15(33 \%)$ & $24(12 \%)$ & & \\
$\mathrm{Au}$ & 150 & $31(69 \%)$ & $70(34 \%)$ & 45.2 & 208 \\
$\mathrm{Ag}$ & 86 & $46(73 \%)$ & $105(30 \%)$ & 63.0 & 346 \\
\hline \hline
\end{tabular}

${ }^{\mathrm{a}}$ Reference 8. 


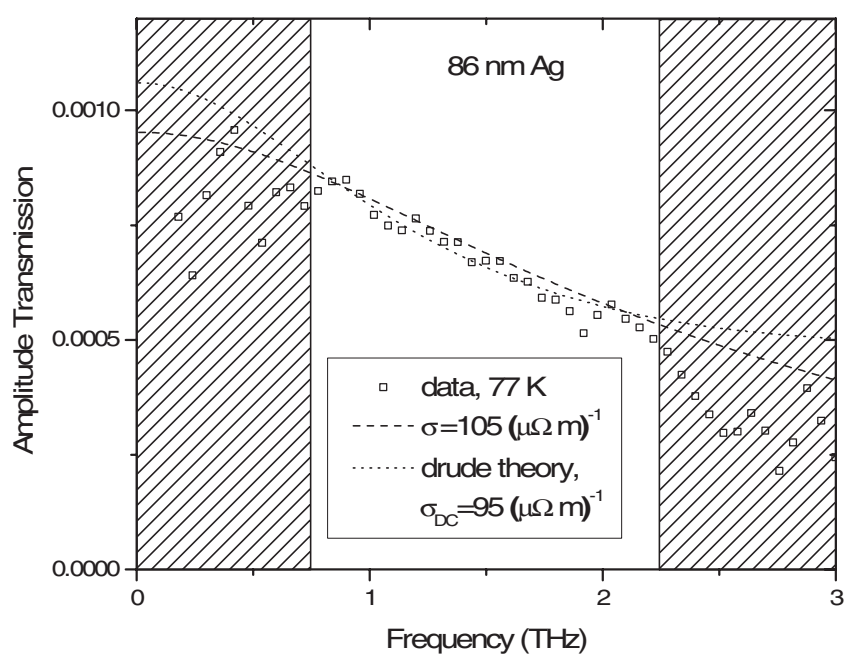

FIG. 5. Amplitude transmission $t$ through $86 \mathrm{~nm} \mathrm{Ag}$ film at $77 \mathrm{~K}$, with different models: frequency independent conductivity and Drude model.

formula $\sigma=i \varepsilon_{0} \omega_{p}^{2} /(2 \pi f+i \Gamma)=\sigma_{\mathrm{dc}}(i \Gamma) /(2 \pi f+i \Gamma)$, where $\sigma_{\mathrm{dc}}$ $=\varepsilon_{0} \omega_{p}^{2} / \Gamma$ is the dc conductivity and $\Gamma$ is the electron scattering rate. The conductivity is reduced as the frequency $f$ approaches the scattering rate $\Gamma / 2 \pi$. One can estimate $\Gamma / 2 \pi$ by scaling the bulk value ${ }^{12}$ inversely by the same ratio of the measured $\sigma$ to the bulk $\sigma$. These estimated values of $\Gamma / 2 \pi$ for the $\mathrm{Al}$ films range from 15 to $46 \mathrm{THz}$, resulting in only a very small difference from the frequency independent conductivity. For the $\mathrm{Au}$ films, the estimated values of $\Gamma / 2 \pi$ range from 3.4 to $16 \mathrm{THz}$, resulting in a minor difference in the shape of the frequency dependent transmission.

However, for the Ag film conductivity at $77 \mathrm{~K}, \Gamma / 2 \pi$ $=2.4 \mathrm{THz}$. This results in a predicted reduction in the conductivity at higher frequencies, resulting in a larger transmission. The measured transmission of the $86 \mathrm{~nm} \mathrm{Ag}$ film at 77 $\mathrm{K}$ is compared in Fig. 5 to the predicted transmission given both a model with a frequency independent conductivity and the Drude model. Despite the low random error, there is a strong possibility of systematic error for transmission amplitudes below 0.0005 . Consequently, we cannot distinguish between a frequency independent conductivity and the Drude model, which both give acceptable fits between 0.75 and $2.25 \mathrm{THz}$.

For scattering rates that are much higher than the considered frequency, the Drude model conductivity is essentially constant and given by $\sigma_{\mathrm{dc}}=\varepsilon_{0} \omega_{p}^{2} / \Gamma$. According to Matthiessen's rule, the total scattering $\Gamma=\Gamma_{P}+\Gamma_{D}$ is the sum of a temperature dependent term $\Gamma_{P}$, dominated by phonons, and a temperature independent term $\Gamma_{D}$, dominated by defects such as grain boundaries. Typically, the scattering of high quality bulk material is dominated by $\Gamma_{P}$ at room temperature. Upon cooling, $\Gamma_{P}$ can be reduced until the scattering is dominated by $\Gamma_{D}$. For lower quality materials, $\Gamma_{D}$ can be a strong component of scattering even at room temperature.
For example, the scattering of bulk $\mathrm{Al}$ at $295 \mathrm{~K}$ is $\Gamma_{P} / 2 \pi$ $\sim \Gamma / 2 \pi=20 \mathrm{THz} .{ }^{12}$ However, the scattering of the $88 \mathrm{~nm}$ thin film at $77 \mathrm{~K}$ as estimated from the measured conductivity is $\Gamma_{D} / 2 \pi \sim \Gamma / 2 \pi=29 \mathrm{THz}$. Assuming $\Gamma_{P}$ for the film is the same as for bulk, the scattering of the film at $295 \mathrm{~K}$ should be $\Gamma / 2 \pi=\Gamma_{P} / 2 \pi+\Gamma_{D} / 2 \pi=49 \mathrm{THz}$. This agrees reasonably well with the estimated $\Gamma$ from the measured $295 \mathrm{~K}$ conductivity of $\Gamma / 2 \pi=46 \mathrm{THz}$. Reasonably good agreement is also obtained for the $\mathrm{Au}$ and $\mathrm{Ag}$ films. In general, the films with the lower relative conductivities have the highest $\Gamma_{D}$, resulting in only a minor change upon cooling. In contrast, the films with the higher relative conductivities generally have the lowest $\Gamma_{D}$, resulting in a sizable change upon cooling.

In summary, the essentially frequency independent conductivities of $\mathrm{Al}, \mathrm{Au}$, and $\mathrm{Ag}$ thin films have been measured via their $\mathrm{THz}$ transmission from 0.5 to $3 \mathrm{THz}$, and found to be much less than their bulk values, particularly at cryogenic temperatures. Our results are consistent with additional scattering mechanisms such as grain boundaries being more important with thin films. These results strongly impact electronic design and metallic cavities and waveguides at these frequencies. The Ag film had much higher conductivity than $\mathrm{Al}$ and $\mathrm{Au}$ films of the same thickness, which may be technologically important. These results are of particular importance to research studies with thin metal film $\mathrm{THz}$ surface plasmons $^{13-15}$ and metamaterials. ${ }^{16}$

This work was partially supported by the National Science Foundation.

${ }^{1}$ N. Laman and D. Grischkowsky, Appl. Phys. Lett. 90, 122115 (2007).

${ }^{2}$ D. Grischkowsky, S. Keiding, M. van Exter, and Ch. Fattinger, J. Opt. Soc. Am. B 7, 2006 (1990).

${ }^{3}$ M. van Exter and D. Grischkowsky, IEEE Trans. Microwave Theory Tech. 38, 1684 (1990).

${ }^{4} \mathrm{M}$. Born and E. Wolf, Principles of Optics, 7th ed. (Cambridge University Press, Cambridge, England, 1999), p. 756.

${ }^{5}$ J. Dai, J. Zhang, W. Zhang, and D. Grischkowsky, J. Opt. Soc. Am. B 21 , 1379 (2004).

${ }^{6}$ Y. Zhao and D. R. Grischkowsky, IEEE Trans. Microwave Theory Tech. $\mathbf{5 5}, 656$ (2007).

${ }^{7}$ S. Ramo, J. R. Whinnery, and T. van Duzer, Fields and Waves in Communications Electronics (Wiley, New York, 1994).

${ }^{8}$ CRC Handbook of Chemistry and Physics, $73 \mathrm{rd}$ ed., edited by D. R. Lide (CRC, Boca Raton, FL, 1992), pp. 12-34.

${ }^{9}$ J. W. C. de Vries, Thin Solid Films 167, 25 (1988).

${ }^{10}$ J. R. Sambles, K. C. Elsom, and D. J. Jarvis, Philos. Trans. R. Soc. London, Ser. A 304, 365 (1982).

${ }^{11}$ M. Walther, D. G. Cooke, C. Sherstan, M. Hajar, M. R. Freeman, and F. A. Hegmann, Phys. Rev. B 76, 125408 (2007).

${ }^{12}$ N. W. Ashcroft and N. D. Mermin, Solid State Physics (Saunders, Philadelphia, 1976), Chap. 1.

${ }^{13}$ D. Qu, D. Grischkowsky, and W. Zhang, Opt. Lett. 29, 896 (2004).

${ }^{14}$ D. Qu and D. Grischkowsky, Phys. Rev. Lett. 93, 196804 (2004).

${ }^{15}$ A. K. Azad and W. Zhang, Opt. Lett. 30, 2945 (2005).

${ }^{16}$ R. Singh, E. Smirnova, A. J. Taylor, J. F. O'Hara, and W. Zhang, Opt. Express 16, 6537 (2008). 